\title{
Fonsecaea spp. in a Case of Corneal Ulcer - A Case Report from a Tertiary Care Hospital, New Delhi
}

\author{
Malhotra $\mathrm{S}^{1 *}$, Dhundial $\mathrm{R}^{1}$, Jerum $\mathrm{H}^{1}$, Bhatia $\mathrm{NJK}^{1}$, Chauhan $\mathrm{A}^{1}$, Jain \\ $\mathrm{S}^{2}$ and Duggal $\mathrm{N}^{1}$ \\ ${ }^{1}$ Department of Microbiology, PGIMER and associated Dr RML Hospital, India \\ 2Department of Ophthalmology, PGIMER and associated Dr RML Hospital, India
}

\section{Case Report}

Volume 2 Issue 4

Received Date: October 14, 2017

Published Date: November 15, 2017

*Corresponding author: Shalini Malhotra, Department of Microbiology, PGIMER and associated Dr RML Hospital, New Delhi, India, E-mail: drshalinimalhotra@yahoo.com

\begin{abstract}
Despite F. pedrosoii being the most common agent of cutaneous blastomycosis, there are few reports of ocular infection by this agent in the literature. This article describes an unusual case of keratitis due to Fonsecaea pedrosoi, which developed after corneal trauma. The patient was a 35-year-old man who presented with a corneal ulcer in the right eye, after sustaining an injury caused by raw vegetable matter. Corneal scrapings were collected for smear and culture examinations. Dematiaceous hyphae were seen on wet mounts of the scrapings, and dark pigmented colonies grew on the culture media. Isolate was identified as Fonsacea pedrosoi.
\end{abstract}

Keywords: Chromoblastomycosis; Dematiaceous fungus; Fonsacea pedrosoi; Antifungals; Keratitis

Abbreviations: KOH: Potassium Hydroxide; SDA: Sabouraud Dextrose Agar; PL: Perception of Light; LPCB: Lactophenol Cotton Blue

\section{Introduction}

The commonest causative agent of chromoblastomycosis is Fonsecaea pedrosoi, which is a dematiaceous fungus [1]. This mainly occurs in the humid tropical regions. Fungal infection of cornea primarily affecting the corneal epithelium and stroma are known as mycotic keratitis which in severe cases can involve endothelium and anterior chamber of eye [2]. Corneal infection due to Fonsecaea pedrosoi is very rare and a few cases of infection are reported in the literature [3-6].
Treatment of this mycosis is challenging not only because of limited antifungals but also due to the need for the prolonged periods of treatment, either with medications or surgery. This paper describes a case of keratitis due to Fonsecaea pedrosoi, a primary cause of cutaneous chromoblastomycosis, but a rarely reported etiology in fungal keratitis.

\section{Case Report}

A 35-year-old agriculture worker presented with complaints of pain, redness, irritation, watering, and photophobia in the right eye of 20 days duration, following trauma by raw vegetable matter. On slit lamp examination, the patient was found to have a dirty white 


\section{Open Access Journal of Ophthalmology}

corneal ulcer $(5 \times 6 \mathrm{~mm})$ with feathery margins, stromal infiltration and hypopyon $(1 \mathrm{~mm})$. There were no satellite lesions; the visual acuity in this eye was "perception of light" (PL) only. The visual acuity in the patient's left eye was 6/18 with no other detectable abnormality. Under local anaesthesia (4\% lignocaine), multiple scrapings were obtained from the base and edges of the corneal ulcer with a sterile blunt cataract knife. A potassium hydroxide (KOH) wet mount and Gram-stained smear of the scrapings were prepared for direct microscopic examination. Scrapings were also inoculated on Sabouraud Dextrose agar (SDA) and blood agar, which were incubated at $25^{\circ} \mathrm{C}$ and $37^{\circ} \mathrm{C}$ respectively. Direct microscopic examination of the $\mathrm{KOH}$ wet mount revealed septate fungal hyphae in the scraped material morphologically suggestive of pheoid fungus. After seven days of incubation SDA showed restricted heaped, brittle colonies dark green to brown in colour with short aerial mycelia. Colonies were folded, velvety to cottony in texture at $25^{\circ} \mathrm{C}$. The colonies matured in the next week and became olivaceous to brown-black in color. (Figure 1) LPCB (lactophenol blue mount) revealed that the mycelium was composed of branching septate dark brown hyphae with short chains of elliptical conidia formed at the ends of conidiophores (Cladosporium like condition) that were branched at apices. The conidia (1.5$3.0 \times 2.5-6.0 \mu \mathrm{m}$ ) were brown and barrel-shaped. (Figure 2) Isolate was identified as F. pedrosoi.

Patient was treated with penetrating keratoplasty and topical amphotericin B $(0.5 \%)$ every hour along with the oral ketoconazole. After 4 months the treatment was discontinued because clinical healing occurred. No relapse of infection was observed three months after the cessation of therapy.

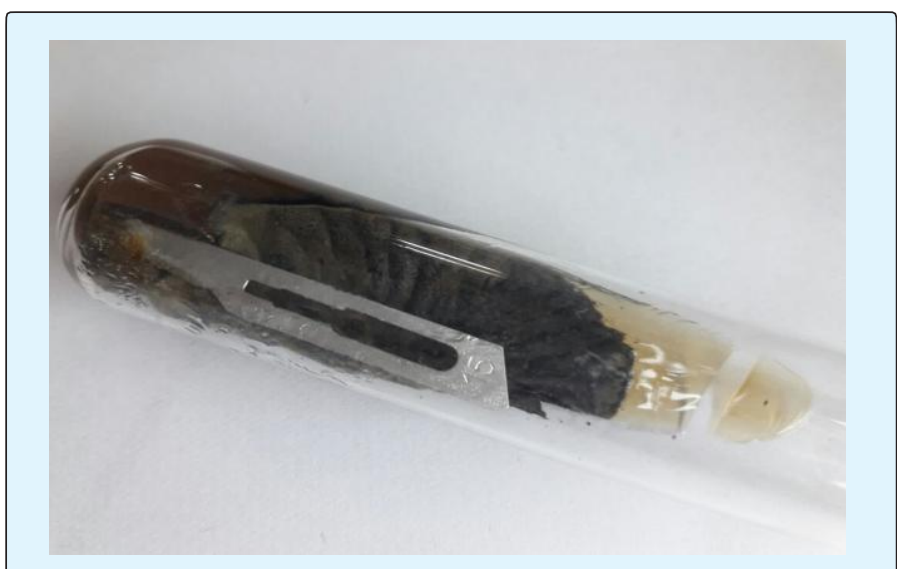

Figure 1: SDA slant showing growth of Fonsecacea spp.

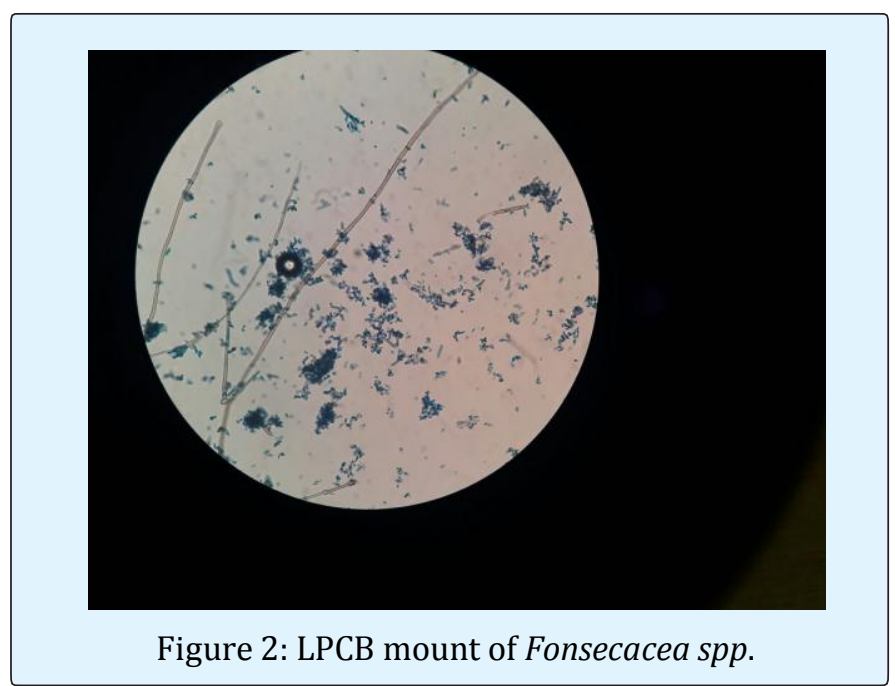

\section{Discussion}

Mycotic keratitis is an important ophthalmologic problem, particularly in rural and outdoor workers [7]. The incidence of ocular fungal infections has increased in the last few years due to the improvement in microbiologic diagnostic techniques and because of introduction of new therapeutic measures such as widespread use of broad-spectrum antibiotics, immunosuppressive drugs and corticosteroids [8].

More than 50 genera of fungi have been implicated in its etiology [9]. Most of these are saprobic organisms that cause opportunistic corneal infections in traumatized or immunologically compromised individuals. Fonsecaea Pedrosoi is a dematiaceous fungi and a primary etiological agent for cutaneous chromoblastomycosis. In India, very few cases of corneal chromoblastomycosis were reported in literature including one case series on F. pedrosoii [3$6,10]$. Men are involved more commonly than women, and agricultural workers and outdoor manual labourers constituted the largest affected occupational group as seen in our case [11,12]. The reason being that saprophytic fungal agents can be traumatically implanted into the cornea to which agricultural workers are more commonly exposed. In our patient, history of ocular trauma with vegetable matter suggested the possibility of fungal infection.

Mycotic keratitis is often associated with considerable morbidity and in severe cases can even lead to end ophthalmitis [13]. Prompt and proper treatment is the best predictors of positive patient outcome. Due to diversity in the causative agents of mycotic keratitis and various dematiaceous fungi involved in such infections, 


\section{Open Access Journal of Ophthalmology}

F.pedrosoi although rare should be considered as one of the important etiological agents. Prompt diagnosis and intensive treatment can prevent the considerable morbidity associated with the disease.

\section{Conclusion}

The key element in the diagnosis of mycotic keratitis is the clinical suspicion by the ophthalmologists and laboratory confirmation of the fungus before prescribing corticosteroids and antibacterial antibiotics. Therefore, precise identification of the causative fungus and institution of appropriate treatment strategy could save the eye from this preventable cause of blindness.

\section{References}

1. Khan S, Kumar A, Vinod V, Prabhakar V, Eapen M, et al. (2015) Chromoblastomycosis due to Fonsecaea pedrosoi: an old wine in a rare bottle. J Infect Dev Ctries 9(3): 325-329.

2. Tuli SS (2011) Fungal keratitis. Clin Ophthalmol 5: 275-279.

3. Barton K, Miller D, Pflugfelder SC (1997) Corneal chromoblastomycosis. Cornea 16(2): 235-239.

4. Kim YS, Song YS, Kim JC (2003) Fungal keratitis caused by Chromomycetes. J Korean Ophthalmol Soc 44(3): 755-759.

5. Höfling-Lima AL, Guarro J, Freitas Dd, Godoy P, Gené J, et al. (2005) Clinical treatment of corneal infection due to Fonsecaea pedrosoi--case report. Arq Bras Oftalmol 68(2): 270-272.
6. Sangwan J, Lathawal S, Juyal D, Sharma N (2013) Fonsecaea pedrosoi: a rare etiology in fungal keratitis. J Clin Diagn Res 7(10): 2272-2273.

7. Srinivasan M, Gonzales CA, Georghe C, Cevallos V, Masearenhas JM, et al. (1997) Epidemiology and etiological diagnosis of corneal ulceration in Madurai, South India. Br J Opthalomol 81(11): 965-71.

8. Prats CH, Tello FL, Jose ABS, Otaolaurruchi JS, Baines JP (2004) Voriconazole in fungal keratitis caused by Scedoporium apiospermum. Ann Pharmacol 38(3): 414-7.

9. Thomas PA (2003) Current perspectives on ophthalmic mycoses. Clin Microbiol Rev 16(4): 73097.

10. Chander J, Sharma A (1994) Prevalence of fungal corneal ulcers in northern India. Infection 22(3): 207209.

11. Kulshareshta OP, Bhargava S, Dutta MK (1973) Keratomycosis - A report of 23 cases. Indian J Ophthal 21(2): 51-55.

12. Dasgupta LR, Gupta AK, Ghosh Roy B, Sunderraj T, Ramamurthy S, et al. (1973) Mycological studies in Keratitis. Indian J Med Res 61(2): 165-168.

13. Esterre P, Andriantsimahavandy A, Ramarcel ER, Pecarrere JL (1996) Forty years of chromoblastomycosis in Madagascar: a review. Am J Trop Med Hyg 55(1): 45-47. 